\title{
Nutritional management of captive cheetahs; is the domestic cat an appropriate model?
}

\author{
$\mathrm{K} \mathrm{Bell}^{1,2}$ \\ ${ }^{1}$ WALTHAM Centre for Pet Nutrition, Waltham-on-the-Wolds, Leicestershire, United Kingdom \\ ${ }^{2}$ Cheetah Outreach, Cape Town, South Africa \\ Email: acinonyxkat@yahoo.com
}

The nutritional requirements of captive cheetahs (Acinonyx jubatus) are unknown, and dietary regimes employed by zoological institutions are typically based on domestic cat (Felis catus) requirements and/or field studies of free-ranging cheetahs. Where data is lacking in regards to the specific nutrient requirements for cheetahs, information from studies in the domestic cat is often extrapolated to the cheetah. A review of the nutritional and reproductive anatomy and physiology of cheetahs and domestic cats was undertaken in order to evaluate the use of the domestic cat as a model species for the captive cheetah. Evaluation of reported anatomical features of domestic cats and cheetahs revealed that, relative to body weight, the internal organs of both species are remarkably similar. In fact, the cheetah appears to bear closer resemblance to the domestic cat in this regard than it does to other large felid species. The shared carnivory and highly conserved genome of the Felidae also appears to have resulted in a number of analogous nutritional metabolic pathways. For example, there is evidence that the cheetah and domestic cat possess equivalent metabolic activity for essential fatty acids, phenols, and xenobiotics. However important differences were detected in values reported for maternal milk composition, vitamin A transport in the serum, as well as growth and developmental features.

A series of experiments were conducted which enabled the investigation of potential inter-specific differences in cheetah growth, the efficacy of using milk replacers formulated for use in domestic cats to hand-rear cheetah cubs, as well as the serum metabolites produced following exposure to a dietary isoflavone. In the first study, the nutrient composition of two milk replacers (one from South Africa, and one from North America) which are used in hand-rearing cheetah cubs was determined. These diets were fed to cheetah cubs along with an indigestible marker (titanium dioxide) during a 14-day feeding trial. Faeces from cubs consuming each of these diets ( $n=4$ on formula 1 , and $n=2$ on formula 2) was collected on the final day. Faeces were then analysed for crude protein, fat, amino acids and dry matter in order to calculate the digestibility of each nutrient. Mean apparent faecal digestibility for both formulas was $>90 \%$ for all nutrients analysed. However, the total crude fat content of both formulas was lower than reported for maternal cheetah milk and both formulas were deficient in at least one of the essential fatty acids $\alpha$-linolenic, linolenic and/or arachidonic acid. Both formulas were low in the majority of essential amino acids and one formula contained an excessive carbohydrate fraction, at the expensive of its protein content. Where data was lacking for cheetah maternal milk, comparison with domestic cat milk revealed excess concentrations of a number of minerals $(\mathrm{K}, \mathrm{Fe}, \mathrm{Zn}$ and $\mathrm{Cu})$, while vitamin $\mathrm{D}_{3}$ was not detected in one formula and only present in concentrations below the minimum requirement for domestic cats in the second formula. Therefore, despite their apparently high digestibility, neither formula was complete or balanced relative to maternal cheetah milk, and/or the requirements established for domestic cats. The results of these dietary analyses indicate that these milk replacers may not provide optimal nutrition for growth in cheetah cubs when used for extended periods. Of particular concern is the finding that these formulas were deficient in one or more essential nutrient, such as vitamin $\mathrm{D}_{3}$ and arachidonic acid.

In a further study, daily body weight, feed and energy intake data was collected from 18 hand-reared cheetah cubs. Growth was approximately linear $\left(\mathrm{R}^{2}=0.95\right)$ prior to weaning, but over the entire age range it exhibited a sigmoidal shape with an asymptotic plateau averaging $57 \mathrm{~kg}$. Energy intake associated with the pre-weaning rate of growth was approximately twice the basal energy requirement calculated from mammalian energetic equations. Regression analysis determined a relationship between metabolic body weight, daily weight gain and metabolisable energy intake, which may be useful in predicting energy intake requirements for hand-reared cheetah cubs. However, comparison with an equation developed previously for suckling kittens indicated that maintenance energy requirements in the cheetah may be proportionally higher than the domestic cat, while the energy for gain factor calculated for cheetah cubs was lower than reported for the domestic cat at a similar age.

In a third study, 4 captive adult cheetahs and 6 adult domestic cats were provided with a single oral bolus of the isoflavones genistein and daidzein. These isoflavones are found naturally in some commercially prepared diets used to feed captive cheetahs in international facilities, but are known to have biological activity in other species (including perturbation of the reproductive system). Five juvenile cheetahs were also included in the study since the milk replacer they were consuming was found to contain these isoflavones. Pharmacokinetic analysis revealed that both species absorbed and excreted isoflavones in the urine and faeces, and both are capable of metabolising genistein and daidzein to alternative forms in the blood, which may represent de-toxification. However, the capacity of the cheetah to conjugate genistein and daidzein appears lower than that of the domestic cat. Furthermore, conjugation appeared reduced in juvenile cheetahs, which may result in greater susceptibility to isoflavone-induced physiological changes.

These findings indicate that differences in nutrition and physiology exist between the domestic cat and cheetah, and as such care must be taken when attempting to use the domestic cat as a model for the cheetah. In general, the assumption that nutritional guidelines developed for the domestic cat are applicable to the nutrition of captive cheetahs requires further validation. While a number of similarities exist between adult domestic cats and cheetahs, many nutrients or metabolic pathways are yet to be investigated. Likewise, the similarities identified between the cheetah and domestic cat cannot necessarily be extended to the rest of the Felidae family. Importantly, the domestic cat does not appear to provide an appropriate model for growth in cheetahs. 